\title{
The Role of Quark Mass in Cold and Dense Perturbative QCD
}

\author{
Eduardo S. FragA ${ }^{1}$ and Paul Romatschke ${ }^{2}$ \\ ${ }^{1}$ Instituto de Física, Universidade Federal do Rio de Janeiro \\ C.P. 68528, Rio de Janeiro, RJ 21941-972, Brazil \\ ${ }^{2}$ Fakultät für Physik, Universität Bielefeld, D-33615 Bielefeld, Germany
}

(Dated: October 24, 2018)

\begin{abstract}
We consider the equation of state of QCD at high density and zero temperature in perturbation theory to first order in the coupling constant $\alpha_{s}$. We compute the thermodynamic potential including the effect of a non-vanishing mass for the strange quark and show that corrections are sizable. Renormalization group running of the coupling and the strange quark mass plays a crucial role. The structure of quark stars is dramatically modified.
\end{abstract}

The investigation of the equation of state for cold and dense strongly interacting matter and its consequences for the possible phases of quantum chromodynamics (QCD) is still in its infancy. From the observational point of view, compact stars provide the most promising "laboratory", since their central densities might be high enough to allow for the presence of deconfined quark matter. These dense objects are usually called quark stars or hybrid stars [1], and some observables, such as their mass-radius relation, may be used to constrain the equation of state for strong interactions.

In this Rapid Communication we compute the thermodynamic potential for cold quark matter with two light (massless) flavors, corresponding to the up and down quarks, and one massive flavor, corresponding to the strange quark, in perturbation theory to first order in $\alpha_{s}$ in the $\overline{\mathrm{MS}}$ scheme. In this fashion, we can easily include modern renormalization group running effects for both the coupling and the strange quark mass. We find that the corrections due to the running nonzero mass are sizable, and should not be neglected in the evaluation of thermodynamic quantities. Solving the TolmanOppenheimer-Volkov (TOV) equations using our equation of state in the presence of electrons, we show that a running strange quark mass dramatically modifies the mass-radius diagram for quark stars, even at first order in $\alpha_{s}$. Although the numbers we present for the mass and radius of quark stars will be modified in a more realistic description, our results are consistent with constraints on the mass-to-radius ratio coming from measurements of gravitationally redshifted absorption lines in the X-ray burst spectra of the low-mass binary EXO0748 - 676 [2].

The thermodynamic potential for cold QCD in perturbation theory to $\sim \alpha_{s}^{2}$ was first computed a long time ago $3,[4,5]$. Nevertheless, the original approach to quark stars 7, 8] made use of the bag model with corrections $\sim \alpha_{s}$ from perturbative QCD to compute the thermodynamic potential 22]. In the massless case, first-order corrections cancel out in the equation of state, so that one ends up with a free gas of quarks modified only by a bag constant. Finite quark mass effects were then estimated to modify the equation of state by less than $5 \%$ and were essentially ignored.
A few years ago, corrections $\sim \alpha_{s}^{2}$ with a modern definition of the running coupling constant were used to model the non-ideality in the equation of state for cold, dense QCD with massless quarks [10]. A perturbative calculation of the thermodynamic potential necessarily produces an unknown scale, $\bar{\Lambda}$, associated with the subtraction point for renormalization. In the case of zero temperature and mass and nonzero chemical potential, $\bar{\Lambda}$ is proportional to the quark chemical potential, $\mu$. Nevertheless, on very general grounds, one can argue that reasonable values for $\bar{\Lambda} / \mu$ lie between 2 and 3 , if one takes perturbative QCD as a model for the equation of state [10] for cold strongly interacting matter. The former value corresponds to a strong first-order chiral phase transition, and the latter to a weak transition or a crossover. In the case of a strong first-order transition it was shown that there might be a new class of compact stars, besides the usual neutron stars, being smaller and much denser, with a large fraction of deconfined quark matter 10, 11]. This approach can be compared to treatments that resort to resummation methods and quasiparticle model descriptions 12, 13, 14, 15]. Remarkably, these different frameworks seem to agree reasonably well for $\mu>>1 \mathrm{GeV}$, and point in the same direction even for $\mu \sim 1 \mathrm{GeV}$ and smaller, where one is clearly pushing perturbative QCD far below its region of applicability.

Even the most recent QCD approaches mentioned above generally neglected quark masses and the presence of a color superconducting gap as compared to the typical scale for the chemical potential in the interior of compact stars, $\sim 400 \mathrm{MeV}$ and higher. However, it was recently argued that both effects should matter in the lower-density sector of the equation of state [16. In fact, although quarks are essentially massless in the core of quark stars, the mass of the strange quark runs up, and becomes comparable to the typical scale for the chemical potential, as one approaches the surface of the star.

In what follows, we present an exploratory analysis of the effects of a finite mass for the strange quark on the equation of state for perturbative QCD at high density, leaving the inclusion of a color superconducting gap in this framework for future investigations. To illustrate the effects and study the modifications in the structure 
of quark stars, we focus on the simpler case of first-order corrections. Results including full corrections $\sim \alpha_{s}^{2}$, as well as technical details of the calculation at each order and renormalization, will be presented in a longer publication [18].

The leading-order piece of the thermodynamic potential of QCD for one massive flavor is given by $[3,4,5]$

$$
\Omega^{(0)}=-\frac{N_{c}}{12 \pi^{2}}\left[\mu u\left(\mu^{2}-\frac{5}{2} m^{2}\right)+\frac{3}{2} m^{4} \ln \left(\frac{\mu+u}{m}\right)\right]
$$

where $N_{c}$ is the number of colors and $u \equiv \sqrt{\mu^{2}-m^{2}}$.

The first-order correction to Eq. (11), also known as the exchange energy $[3,4,5]$, has the form

$$
\begin{aligned}
\Omega^{(1)}= & -2 \pi \alpha_{s} \frac{N_{c}^{2}-1}{2} \mathcal{F}_{P, Q, K} \delta(P-Q-K) \times \\
& \frac{\operatorname{tr}\left[\gamma_{\mu}\left(\not P+m_{f}\right) \gamma^{\mu}\left(Q+m_{f}\right)\right]}{\left(P^{2}-m_{f}^{2}\right)\left(Q^{2}-m_{f}^{2}\right) K^{2}} .
\end{aligned}
$$

We work in the Feynman gauge and have adopted the abbreviation

$$
\mathcal{Y}_{P} \equiv T \sum_{p_{0}} \int \frac{d^{3} p}{(2 \pi)^{3}}
$$

and $\delta(P-Q)=\beta \delta_{p_{0}, q_{0}}(2 \pi)^{3} \delta^{3}(\vec{p}-\vec{q})$, with temperature $T=1 / \beta$. Here $p_{0}=(2 n+1) i \pi T+\mu$ is the frequency of the quark having four-momentum $P$ and a chemical potential $\mu$, and the sum is understood to be carried out over the integers $n[\underline{6}$.

Using standard quantum field theoretical methods, one obtains the complete renormalized exchange energy for a massive quark in the $\overline{\mathrm{MS}}$ scheme (in the limit $T \rightarrow 0$ ):

$$
\begin{aligned}
\Omega^{(1)}= & \frac{\alpha_{s}\left(N_{c}^{2}-1\right)}{16 \pi^{3}}\left[3\left(m^{2} \ln \frac{\mu+u}{m}-\mu u\right)^{2}-2 u^{4}\right. \\
& \left.+m^{2}\left(6 \ln \frac{\bar{\Lambda}}{m}+4\right)\left(\mu u-m^{2} \ln \frac{\mu+u}{m}\right)\right]
\end{aligned}
$$

The thermodynamic potential to order $\alpha_{s}$ for one massive flavor, given by the sum of Eqs. (4) and (11), depends on the quark chemical potential $\mu$ and on the renormalization subtraction point $\bar{\Lambda}$ both explicitly and implicitly through the scale dependence of the strong coupling constant $\alpha_{s}(\bar{\Lambda})$ and the mass $m(\bar{\Lambda})$. The scale dependencies of both $\alpha_{s}$ and $m$, which in the following we will take to be the mass of the strange quark, are known up to 4 -loop order in the $\overline{\mathrm{MS}}$ scheme [20]. Since we have only determined the free energy to first order in $\alpha_{s}$, we choose

$$
\begin{aligned}
\alpha_{s}(\bar{\Lambda}) & =\frac{4 \pi}{\beta_{0} L}\left[1-2 \frac{\beta_{1}}{\beta_{0}^{2}} \frac{\ln L}{L}\right], \\
m_{s}(\bar{\Lambda}) & =\hat{m}_{s}\left(\frac{\alpha_{s}}{\pi}\right)^{4 / 9}\left[1+0.895062 \frac{\alpha_{s}}{\pi}\right],
\end{aligned}
$$

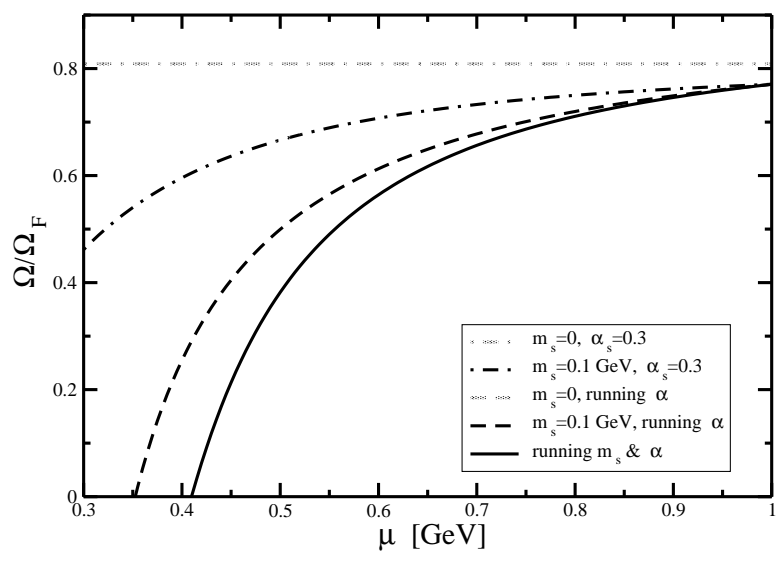

FIG. 1: $\Omega / \Omega_{F}$ at $\bar{\Lambda}=2 \mu$ for fixed coupling (dash-dotted lines), including 2-loop running coupling (dashed lines) and including running of $m_{s}$ (full line). The light gray lines indicate $m_{s}=0$.

where $L=2 \ln \left(\bar{\Lambda} / \Lambda_{\overline{\mathrm{MS}}}\right), \beta_{0}=11-2 N_{f} / 3$, and $\beta_{1}=$ $51-19 N_{f} / 3$ and we take $N_{f}=3$. The scale $\Lambda_{\overline{\mathrm{MS}}}$ and the invariant mass $\hat{m}_{s}$ are fixed by requiring [21] $\alpha_{s} \simeq 0.3$ and $m_{s} \simeq 100 \mathrm{MeV}$ at $\bar{\Lambda}=2 \mathrm{GeV}$; one obtains $\Lambda_{\overline{\mathrm{MS}}} \simeq$ $380 \mathrm{MeV}$ and $\hat{m}_{s} \simeq 262 \mathrm{MeV}$. With these conventions, the only freedom left is the choice of $\bar{\Lambda}$.

Note that Eq.(4) corresponds to an earlier result [17] which was derived in a different renormalization scheme. Sticking consistently to the $\overline{\mathrm{MS}}$ scheme offers however some advantages, e.g. one choice of $\bar{\Lambda}$ allows one to use modern-day restrictions [21] on both the ( $\mu$-dependent) values of the strange quark mass and the strong coupling $\alpha_{s}$, whereas the authors of 17] had to resort to (physically motivated) choices for these. Also, our calculation is straightforward to extend to higher orders, since the coefficients for the renormalization group equations are known up to 4-loop order in the $\overline{\mathrm{MS}}$ scheme [20].

In Fig. 1 we show the thermodynamic potential for one massive flavor (the strange quark), normalized by the free (massless) Fermi result, computed in the cases of fixed and running coupling and strange quark mass for $\bar{\Lambda}=2 \mu$. As one can see, renormalization group improvements bring sizeable corrections in the region relevant for the structure of compact stars, $\mu<1 \mathrm{GeV}$. At $\mu=600 \mathrm{MeV}$, for which $\alpha_{s} \sim 0.43$, including a fixed mass for the strange quark decreases the thermodynamic potential by $\sim 15 \%$. The running of the mass (which insures renormalization scale invariance of $\Omega$ up to order $\alpha_{s}$ ) brings plus $\sim 8 \%$ of decrease.

To study the effect of the finite strange quark mass on the equation of state for electrically neutral quark matter with 2 light (massless) flavors (up and down quarks) and one massive flavor (strange quark), we have to include electrons, with chemical potential $\mu_{e}$. Imposing 
beta equilibrium, one has

$$
\begin{gathered}
d \rightarrow u+e+\bar{\nu}_{e}, \quad u+e \rightarrow d+\nu_{e} \\
s \rightarrow u+e+\bar{\nu}_{e}, \quad u+e \rightarrow s+\nu_{e} \\
s+u \leftrightarrow d+u
\end{gathered}
$$

Since neutrinos are lost rather quickly, one may set their chemical potential to zero [1], so that chemical equilibrium yields

$$
\mu_{d}=\mu_{s}=\mu, \quad \mu_{u}+\mu_{e}=\mu,
$$

with $\mu_{u}, \mu_{d}$ and $\mu_{s}$ the up, down and strange quark chemical potentials, respectively. On the other hand, overall charge neutrality requires

$$
\frac{2}{3} n_{u}-\frac{1}{3} n_{d}-\frac{1}{3} n_{s}-n_{e}=0
$$

where $n_{i}$ is the number density of the particle species $i$. Together, the above equations insure that there is only one independent chemical potential, which we take to be $\mu$. Number densities are determined from the thermodynamic potential by $n_{i}=-(\partial \Omega / \partial \mu)$ and the total energy density is given by $\epsilon=\Omega+\sum_{i} \mu_{i} n_{i}$, where $\Omega=\sum_{i}\left(\Omega_{i}^{(0)}+\Omega_{i}^{(1)}\right)$ and again $i$ refers to the particle species. The pressure $P$ is

$$
P=n_{B} \frac{\partial \epsilon}{\partial n_{B}}-\epsilon,
$$

where $n_{B}=\frac{1}{3}\left(n_{u}+n_{d}+n_{s}\right)$ is the baryon number density and the Gibbs potential per particle is given by $\frac{\partial \epsilon}{\partial n_{B}}=\left(\mu_{u}+\mu_{d}+\mu_{s}\right)$. We restrict the freedom of choice for $\bar{\Lambda}\left(\mu_{u}, \mu_{d}, \mu_{s}\right)$ by requiring that in case of vanishing strange quark mass all quark chemical potentials and number densities become equal so that $P^{\left(m_{s}=0\right)}=$ $-\Omega^{\left(m_{s}=0\right)}$ and, consequently, one has $\mu_{e} \rightarrow 0$ [23]. Furthermore, in order to compare our findings to existing results in the literature [10, 13], we require that in the massless case $\bar{\Lambda}=2 \mu$. Consequently, we choose $\bar{\Lambda}=\frac{2}{3}\left(\mu_{u}+\mu_{d}+\mu_{s}\right)$, but have tested that our results are not much affected by other choices obeying the above conditions [24].

The effects of the finite strange quark mass on the total pressure and energy density for electrically neutral quark matter (plus electrons) are given in Fig. 2 There we show results for 3 light flavors and running coupling, corresponding to the case considered in [10], and for 2 light flavors and one massive flavor, with both running coupling and strange quark mass (which reaches $m_{s} \sim 137$ $\mathrm{MeV}$ at $\mu=500 \mathrm{MeV}$ ).

As can be seen from this Figure, there is a sizable difference between zero and finite strange quark mass pressure and energy density for the values of the chemical potential in the region that is relevant for the physics
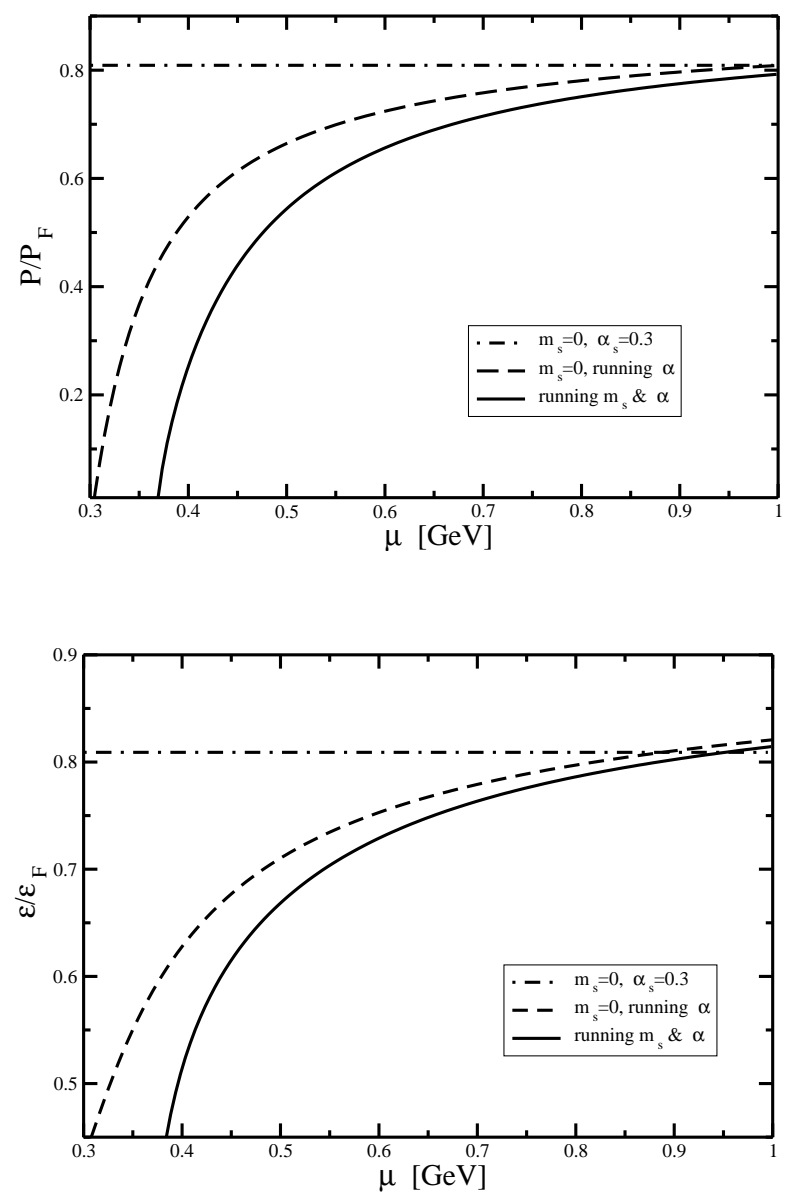

FIG. 2: Pressure and energy density scaled by Fermi values for $\bar{\Lambda}=\frac{2}{3}\left(\mu_{u}+\mu_{d}+\mu_{s}\right)$. We show results without renormalization group improvements (dash-dotted lines), running coupling (dashed lines), both for $m_{s}=0$, and results with running mass and coupling (full lines).

of compact stars, as was already expected from the results presented in Fig. 11 As has been noticed by several authors [11, 12, 16], the resulting equation of state, $\epsilon=\epsilon(P)$, can be approximated by a non-ideal bag model form

$$
\epsilon=4 B_{e f f}+a P
$$

Here $a \sim 3$ is a dimensionless coefficient while $B_{\text {eff }}$ is the effective bag constant of the vacuum. Concentrating on the low-density part of the equation of state, one finds for massless strange quarks the parameters $B_{\text {eff }}^{1 / 4} \simeq 117 \mathrm{MeV}$ and $a \simeq 2.81$ while the inclusion of the running mass raises these values to $B_{\text {eff }}^{1 / 4} \simeq 137 \mathrm{MeV}$ and $a \simeq 3.17$ (all values having been obtained by including a running $\alpha_{s}$ in the equations of state). Therefore, we expect important consequences in the mass-radius relation of quark stars due to the inclusion of a finite mass for the strange quark.

The structure of a quark star is determined by the solution of the TOV equations [1]. One solves TOV by inte- 


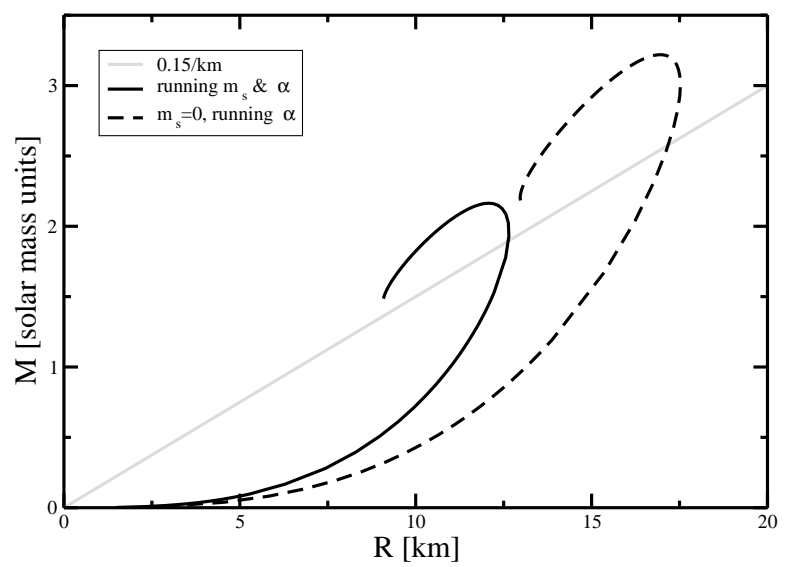

FIG. 3: Mass-radius relation for quark stars based on an equation of state with running mass and coupling (full line) and for massless strange quarks (dashed line), for running coupling and with $\bar{\Lambda}=\frac{2}{3}\left(\mu_{u}+\mu_{d}+\mu_{s}\right)$.

grating from some central starting pressure up to the surface of the star, where the pressure vanishes. The numerical solution of these equations in the case of our equation of state for electrically neutral quark matter gives the mass-radius relation shown in Fig. 3] One can see from the Figure that corrections to the mass and radius of quark stars due to a running strange quark mass can be very large, $\sim 25 \%$. Also, while the most massive star for the $m_{s}=0$ equation of state (with $M / M_{\odot} \simeq 3.2$ and radius $\sim 17 \mathrm{~km}$ ) has a central density of $n_{B} \simeq 0.5 \mathrm{fm}^{-3}$, this number increases to $n_{B} \simeq 0.83 \mathrm{fm}^{-3}$ (at $\mu=470$ $\mathrm{MeV})$ for the heaviest $\operatorname{star}\left(M / M_{\odot} \simeq 2.16\right.$ at $\left.\sim 12 \mathrm{~km}\right)$ of the massive equation of state. One can put constraints on the mass-to-radius ratio by measuring the gravitational redshift at the surface of a neutron star. Our results for the mass-radius relation are consistent with measurements reported by Cottam et al. [2], who obtain a redshift $z=0.35$ for the binary EXO0748 - 676, implying a ratio $\left(M / M_{\odot}\right) / R \sim 0.15 / \mathrm{km}$, also shown in Fig. 3] The inclusion of $\sim \alpha_{s}^{2}$ corrections to the pressure will increase its non-ideality and produce quark stars which are smaller, denser and less massive [10, 11].

We have calculated the thermodynamic potential for cold quark matter with two light flavors and one massive flavor in perturbation theory to first order in $\alpha_{s}$ in the $\overline{\mathrm{MS}}$ scheme. Results including full corrections $\sim \alpha_{s}^{2}$ will appear soon [18] and will certainly modify the numbers we present here, since we know that corrections are large for this region of chemical potential [10, 13]. Moreover, for a realistic description of the structure of quark stars, one has to match the high-density equation of state onto a low-density hadronic one. This will also shift the values of masses and radii for the stars and, in some cases, allow for a new class of compact objects. Contributions due to color superconductivity [16] as well as chiral condensa- tion [19] also affect this picture . Finally, if one aims for quantitatively tenable results, also the $\sim \alpha_{s}^{3}$ contributions to the pressure should eventually be calculated. Therefore, our numbers for the maximum mass and radius are meant only to be suggestive, and the point that we want to stress is the size of the corrections coming from the inclusion of a finite strange quark mass.

In this Rapid Communication we present a still rather incomplete picture of cold and dense quark matter and the role it plays in the core of compact stars. Even so, we believe that our main conclusion is qualitatively correct: modifications in the pressure due to the inclusion of a nonzero strange quark mass are sizable and can dramatically modify the structure of quark stars.

We would like to thank D. Bödeker, M. Laine, R.D. Pisarski and A. Rebhan for fruitful discussions. The authors also thank F. Gelis and E. Iancu for their kind hospitality at the Service de Physique Théorique, CEA/Saclay, where part of this work has been done. The work of E.S.F. is partially supported by CAPES, CNPq, FAPERJ and FUJB/UFRJ. P.R. was supported by the DFG-Forschergruppe Bo 1251/2-2 and KA 1198/4-4.

[1] N. K. Glendenning, Compact Stars - Nuclear Physics, Particle Physics, and General Relativity (Springer, New York, 2000); H. Heiselberg and M. Hjorth-Jensen, Phys. Rept. 328, 237 (2000).

[2] J. Cottam, F. Paerels and M. Mendez, Nature 420, 51 (2002).

[3] B. A. Freedman and L. D. McLerran, Phys. Rev. D16, 1130 (1977); ibid., D16, 1147 (1977); ibid., D16, 1169 (1977); ibid., D17, 1109 (1978).

[4] V. Baluni, Phys. Rev. D 17, 2092 (1978).

[5] T. Toimela, Int. J. Theor. Phys. 24, 901 (1985) [Erratumibid. 26, 1021 (1987)].

[6] J.I. Kapusta,Finite-temperature field theory (Cambridge University Press, 1989).

[7] E. Witten, Phys. Rev. D 30, 272 (1984).

[8] C. Alcock, E. Farhi and A. Olinto, Astrophys. J. 310, 261 (1986); P. Haensel, J. L. Zdunik, and R. Schaeffer, Astron. Astrophys. 160, 121 (1986).

[9] A. R. Bodmer, Phys. Rev. D 4, 1601 (1971).

[10] E. S. Fraga, R. D. Pisarski and J. Schaffner-Bielich, Phys. Rev. D 63, 121702 (2001).

[11] E. S. Fraga, R. D. Pisarski and J. Schaffner-Bielich, Nucl. Phys. A 702, 217 (2002).

[12] A. Peshier, B. Kämpfer and G. Soff, Phys. Rev. C61, 045203 (2000); Phys. Rev. D 66, 094003 (2002).

[13] J. P. Blaizot, E. Iancu and A. Rebhan, Phys. Rev. D 63, 065003 (2001).

[14] J. O. Andersen and M. Strickland, Phys. Rev. D 66, 105001 (2002).

[15] A. Rebhan and P. Romatschke, Phys. Rev. D 68, 025022 (2003).

[16] M. Alford and S. Reddy, Phys. Rev. D 67, 074024 (2003); M. Alford, M. Braby, M. Paris and S. Reddy, nucl-th/0411016 
[17] E. Farhi and R. L. Jaffe, Phys. Rev. D 30, 2379 (1984).

[18] E. S. Fraga and P. Romatschke, in preparation.

[19] M. Buballa and M. Oertel, Nucl. Phys. A 703, 770 (2002); S. B. Ruster et al., hep-ph/0503184 D. Blaschke et al., hep-ph/0503194

[20] J. A. M. Vermaseren, S. A. Larin and T. van Ritbergen, Phys. Lett. B 405, 327 (1997).

[21] S. Eidelman et al. [Particle Data Group Collaboration], Phys. Lett. B 592, 1 (2004).

[22] Actually, the authors of [8] considered the structure of strange stars in which, as suggested by Bodmer and Witten [7, 9], strange quark matter would be the true ground state of QCD. In this work, we consider quark stars, so that a realistic description should match our equation for high density QCD onto a hadronic equation of state at lower densities.

[23] This requirement is not automatic since here $\Omega$ is only renormalization scale invariant up to first order in $\alpha_{s}$.

[24] Relaxing these conditions one finds that the renormalization scale dependence of final results is sizeable as will be shown in [18]; however, we want to concentrate on finite mass effects at fixed $\bar{\Lambda}$ in this publication. 